\title{
One injection for a great projection: a quick and simple procedure for nipple reconstruction
}

\author{
Sara Tanini, Sara Calabrese, Elena Lucattelli, Giulia Lo Russo \\ Department of Plastic and Reconstructive Microsurgery, Careggi University Hospital, Florence, Italy
}

\begin{abstract}
Women attach great importance to the presence of a three-dimensional nipple upon completion of the breast reconstruction process. To meet patients' expectations, nipple-areolar complex reconstruction should achieve symmetry in position, size, shape, texture, and color, as well as minimizing donor-site morbidity. However, it is well known that regardless of the reconstructive technique, loss of nipple projection can be reasonably expected. We developed and evaluated a quick, simple, and innovative technique using injectable Integra Flowable Wound Matrix to increase nipple projection after reconstruction. Twenty breast cancer patients who underwent nipple reconstruction resulting in unsatisfactory projection were enrolled in our retrospective study. Nipple projection was measured at the time of surgery and after 6 and 12 months. A visual analogue scale was used to assess patients' satisfaction. Our technique yielded reliable results in terms of the long-lasting maintenance of nipple projection. This method is high-priced, but cost-effective, since one kit may suffice for three patients. Furthermore, our patients were very appreciative of this technique as a single-step, minimally invasive, painless procedure with no reported necessity of re-intervention.
\end{abstract}

Keywords Nipple / Breast / Breast neoplasm / Dermal filler / Acellular dermis

\author{
Correspondence: Giulia Lo Russo \\ Department of Plastic and \\ Reconstructive Microsurgery, Careggi \\ University Hospital, Via Taddeo \\ Alderotti 56, Florence 50139, Italy \\ Tel: $+39-055244950$ \\ Fax: $+39-0557948178$ \\ E-mail: giulialorusso70@gmail.com
}

\section{INTRODUCTION}

Reconstruction of the nipple-areolar complex (NAC) is considered to be the final step of a successful post-mastectomy breast reconstruction. Many studies have pointed out the degree to which the presence of the nipple in a reconstructed female breast has a positive impact on patients' overall satisfaction and quality of life [1]. Furthermore, the little touches that make the reconstructed breast aesthetically pleasing in women's eyes are nipple position and projection in comparison with the contralateral healthy side [2].

Since the 1940s, when the first article on NAC reconstruction was published, many reconstructive attempts have been made over time to optimize the technical aspects of reconstruction, such as nipple symmetry in terms of position, size, shape, texture, color, and projection, while minimizing donor-site morbidity.

To summarize, nipple reconstruction can be performed with grafts, local flaps, or combinations of the two [3]. Even though surgeons can draw upon a plethora of techniques to reconstruct an ideal nipple in terms of shape and position, unfortunately, all extant techniques appear to be quite inefficient regarding nipple protrusion. The ultimate limit of nipple reconstruction, indeed, is currently the maintenance of its projection over time [4]. 
In the present study, we report our preliminary experience with the use of injectable Integra Flowable Wound Matrix (IFWM; Integra LifeSciences Corp., Plainsboro, NJ, USA) to increase and stabilize nipple projection after oncoplastic breast surgery.

\section{IDEA}

Clinical evidence of loss of nipple projection after reconstruction with local flaps or grafts led us to develop a new method of increasing and stabilizing nipple projection over time. We conducted a retrospective study, enrolling 20 patients with a history of breast cancer who underwent nipple reconstruction between February 2017 and April 2018 by means of a nipple graft from the native nipple (seven patients) or of a pure dermal C-V flap (13 patients). In all patients, breast reconstruction with an expander or implant had previously been performed. All the nipple reconstructive procedures resulted in an unsatisfactory projection of the reconstructed nipple (less projection than the contralateral nipple). To overcome this problem, we devised a simple and quick solution that could be performed on an outpatient basis without anesthesia, but could guarantee a stable result over time: the injection of IFWM.

These procedures were conducted in an outpatient setting. IFWM was supplied sterile, in a single-use kit containing a dry granulated collagen syringe pouch, an empty sterile syringe pouch and a luer-lock connector accessory pouch.

IFWM was prepared according to the manufacturer's instructions by dispensing saline fluid into the dry collagen syringe through the luer-lock connector and depressing the plungers back and forth at least 15 times until a gel-like consistency was achieved. A homogeneous mixture was thus obtained under sterile conditions. We suggest greater rehydration to obtain a more fluid solution, which is useful in this type of procedure.

At this stage, on average, $1 \mathrm{~mL}$ of IFWM was injected. Keeping the nipple still between the thumb and forefinger, an 18-gauge needle was threaded through the base of the nipple up to its central point, orientated at $45^{\circ}$ to the major axis of the nipple. The gel-like IFWM was slowly injected until the desired projection was obtained. Due to the gelatinous consistency of the IFWM, it was usually necessary to apply some pressure on the plunger of the syringe. Normally, we injected no more than 1 $\mathrm{mL}$ of IFWM (Fig. 1). We suggest using one kit for each patient as best practice. However, while maintaining strict adherence to sterility rules, it may be possible to divide the initial syringe content into three equal parts of $1 \mathrm{~mL}$ that are transferred to three luer-lock syringes, enabling a single kit to be used for three different patients consecutively. At the end of the surgical proce-

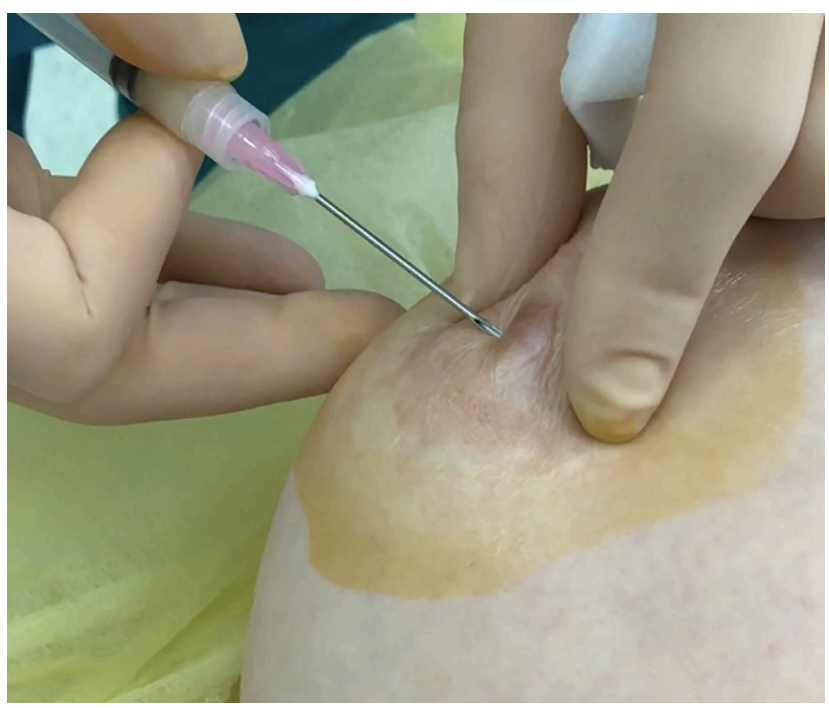

Fig. 1. Surgical procedure. The outpatient procedure involved an injection of approximately $1 \mathrm{~mL}$ of Integra Flowable Wound Matrix without anesthesia through an 18-gauge needle.

dure, Steri-Strips were placed at the basis of the reconstructed nipple and the patients were warned to avoid nipple compression for 3 weeks.

The main evaluation criterion was the measurement of nipple projection, performed before injection, immediately after injection (P0) and 6 months (P6) and 12 months (P12) after the injection.

The secondary endpoints were complications (pain, infection, allergy, necrosis, etc.) and patients' satisfaction, which was rated using a visual analogue scale between 1 and 5 (1, poor; 2, disappointing; 3 , satisfactory; 4 , good; 5 , excellent). The same investigator made all the measurements using a caliper and collected all the data. A statistical analysis of the measurements, including mean values and standard deviations, was performed.

\section{DISCUSSION}

As patients associate the stage of NAC reconstruction with a sense of completeness after oncoplastic breast surgery, many attempts have been made over the decades to refine the plethora of related surgical techniques.

The use of a composite free graft from the labium minus by Adams in 1944 was the first described technique aiming to recreate the original NAC pigment and texture after mastectomy.

Since then, plastic surgeons have tested the use of various composite grafts for nipple reconstruction, harvested from autologous toe pulp, auricular cartilage, rib cartilage, or skin from the superior inner thigh [5]. The first description of the local skin flap technique for nipple reconstruction is attributable to 
Berson in 1949, although local flaps were not widely adopted until the 1980s [5]. Nipple reconstruction by shaving off the top of the contralateral normal nipple dates to 1972 . To date, the most popular methods of nipple reconstruction are subdermal pedicle flaps, raised as full-thickness skin flaps that receive their blood supply from the dermal plexus.

Over time, various flap techniques have been described, including the C-V flap [6], star flap, skate flap, bell flap, top-hat flap, and S flap [4]. As such, there is currently no perfect single surgical strategy for nipple reconstruction to address the challenge of creating a three-dimensional structure from a two-dimensional surface. Flap-based NAC reconstructions continue to be plagued by poor long-term cosmetic outcomes. In particular, the most common issue following nipple reconstruction is postoperative loss of projection, which leads to flattening of the reconstructed nipples.

Although flaps allow surgeons to overbuild the nipple by $25 \%$ to $50 \%$, thereby preempting the ensuing shrinkage, previous studies have shown that the long-term reduction of nipple projection varies between $41 \%$ and $74 \%$ over 6 months with the commonly used techniques, both in autologous and implantbased breast reconstruction. Shestak et al. [7] also reported that when a skate or C-V flap was used, the greatest loss of projection occurred in the first 3 months, followed by stabilization at 6 months. It is estimated that in $10 \%$ to $15 \%$ of reconstructions, the nipple will flatten beyond expectations and need to be recreated.

The causes of postoperative nipple flattening are multifactorial, likely including poor circulation that may lead to flap necro- sis, lack of natural anatomical infrastructure, centrifugal forces under the reconstructed nipple, and wound contracture. In addition, most local flap types primarily rely on fat as both the foundation and major structural support of the recreated nipples. Fat tissue used as underlying structural support for the nipple may undergo necrosis, which might explain the inability of flaps to successfully maintain long-term nipple projection.

The belief that nipple shrinkage is exacerbated by the absence of rigid tissue providing structural support end has motivated the development of current augmented-flap techniques [8].

Some researchers have advocated supplementing reconstructive procedures with various materials such as dermal fat grafts [9], auricular cartilage, rib cartilage, acellular dermal substitute $[10]$, and various dressings in order to maintain the long-term projection of reconstructed nipples. Furthermore, the synthetic materials used to augment nipple projection include poly-tetrafluoroethylene implants, artificial bone, and semi-permanent injectable fillers such as calcium hydroxyapatite [11].

Flaps with autologous graft/alloplastic/allograft augmentation (cartilage, fat, calcium hydroxyapatite, acellular dermal matrix, poly-methylmethacrylate, biologic collagen) have so far shown a minor loss of nipple projection, but may expose the patient to a somewhat increased risk of complete flap necrosis [12].

To overcome these limitations, we present our preliminary experience of nipple reconstruction with local flaps or grafts combined with IFWM, an injectable matrix composed of a granulated cross-linked bovine tendon collagen and glycosaminoglycan. In the form of a gel, it was first thought to enhance the healing outcomes of deep soft tissues or tunneling wounds, such as dia-

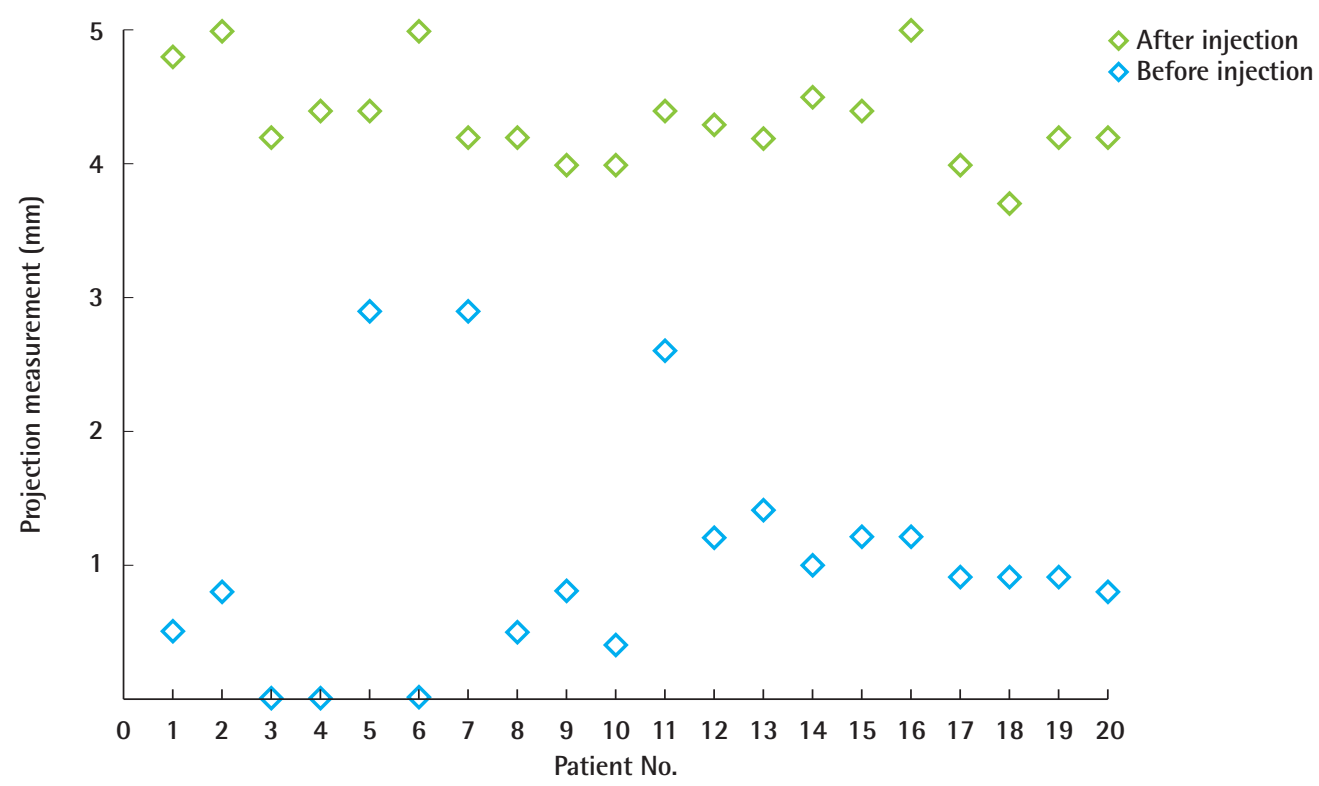

Fig. 2. Trends in nipple projection over time. Change of nipple projection at 12 months after the injection of Integra Flowable Wound Matrix. 
betic ulcers, pressure ulcers, venous ulcers, chronic vascular ulcers [13], and other wounds that may benefit from a flowable dermal core.

The main advantage of IFWM is that the granulated collagenglycosaminoglycan, hydrated with saline solution, provides a scaffold for cellular invasion and capillary growth followed by new tissue deposition. This cellular colonization makes this product long-lasting, as it enables the creation of a rigid infrastructure to support the nipples. Moreover, it is user-friendly and appreciated by patients, since it is painless and injectable under local anesthesia through a thin needle. The only disadvantage of this material is its cost.

We suggest that it is best practice to use one kit for one patient, but to overcome the limitation of cost, it may be possible to di- vide the initial syringe content, while strictly adhering to sterility rules, into three equal parts of $1 \mathrm{~mL}$ that are transferred to three luer-lock syringes for use in three different patients consecutively. This adaptation made our procedure both time- and cost-effective.

IFWM and its progenitor, the Integra Dermal Regeneration Template (IDRT), are not interchangeable. The difference is not in their composition, as both are composed of cross-linked bovine tendon collagen and glycosaminoglycan, but in their physical state. IFWM is sold in a syringe containing granulated and dehydrated components that must therefore be hydrated before injection. IDRT, instead, is solid and is sold in sheets composed of two layers (a thick inner layer made of collagen and glycosaminoglycan and a thin outer layer made of silicone).
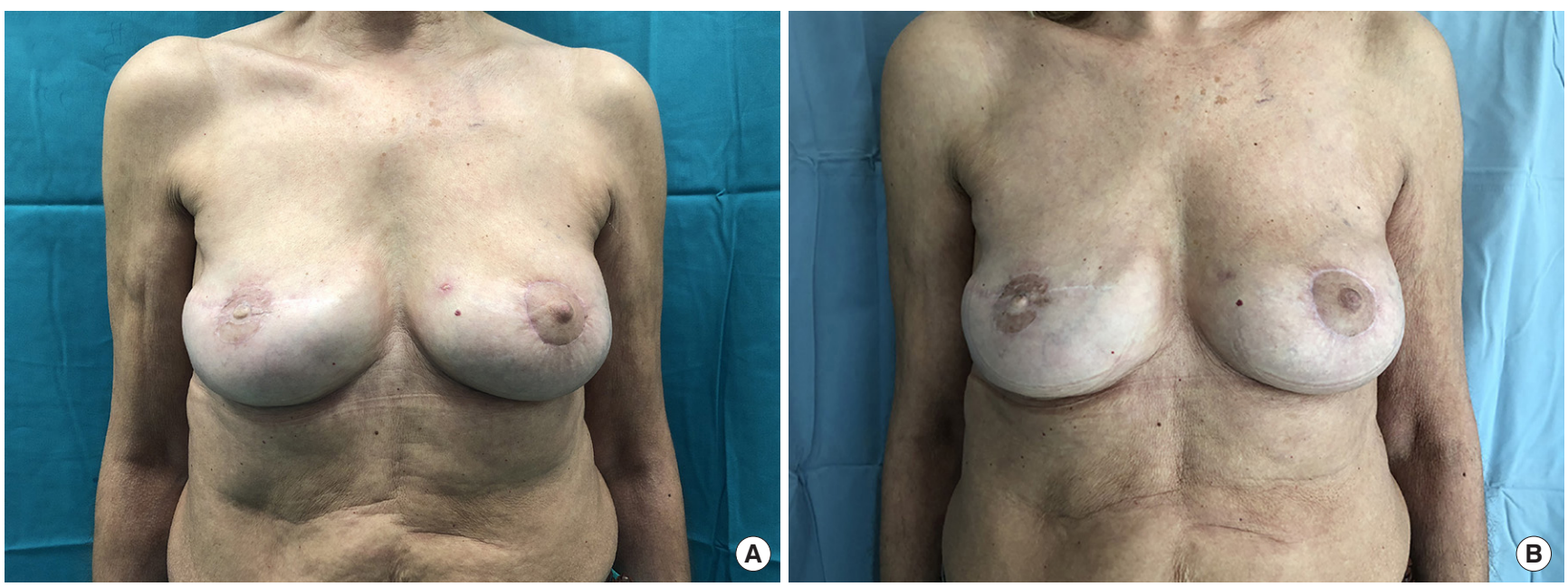

Fig. 3. Nipple reconstruction case with a graft. (A) Preoperative front view of a patient whose right nipple was reconstructed using the nipple sharing technique. (B) Postoperative front view 6 months after the injection of Integra Flowable Wound Matrix into the previously reconstructed right nipple.
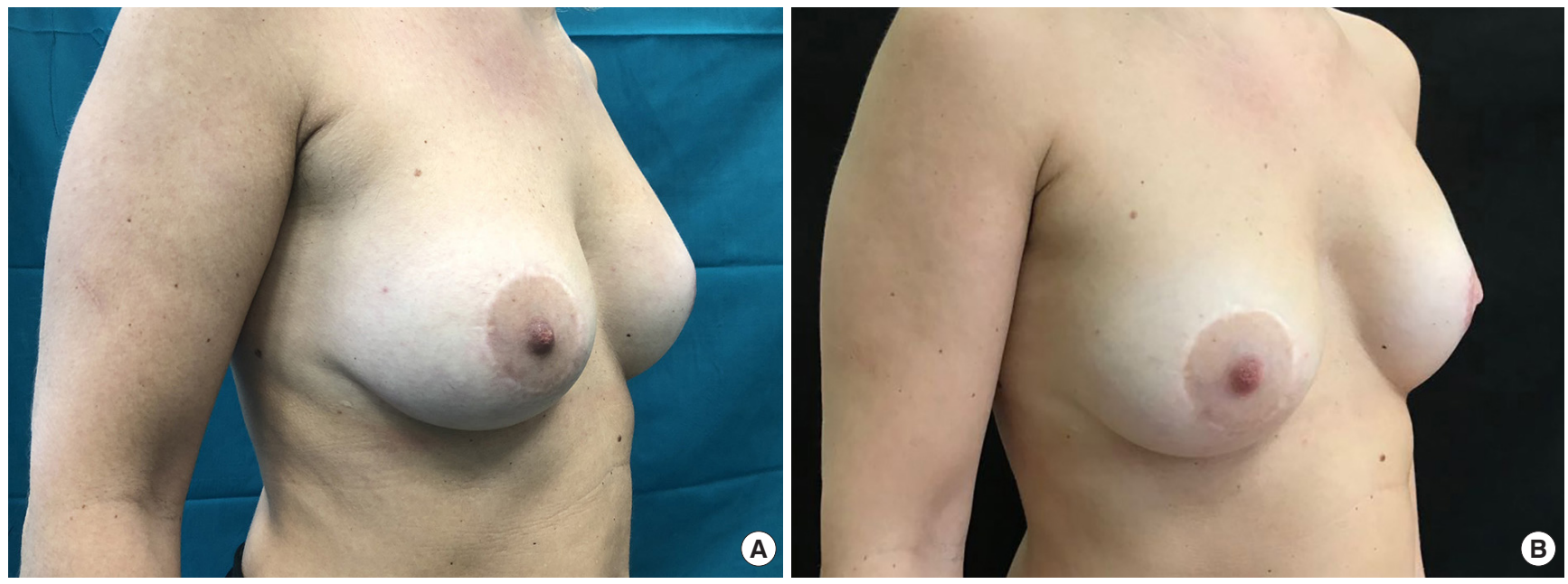

Fig. 4. Nipple reconstruction case with a C-V flap. (A) Preoperative lateral view of a patient whose left nipple was reconstructed with a C-V flap. (B) Postoperative lateral view 6 months after the injection of Integra Flowable Wound Matrix into the previously reconstructed left nipple. 

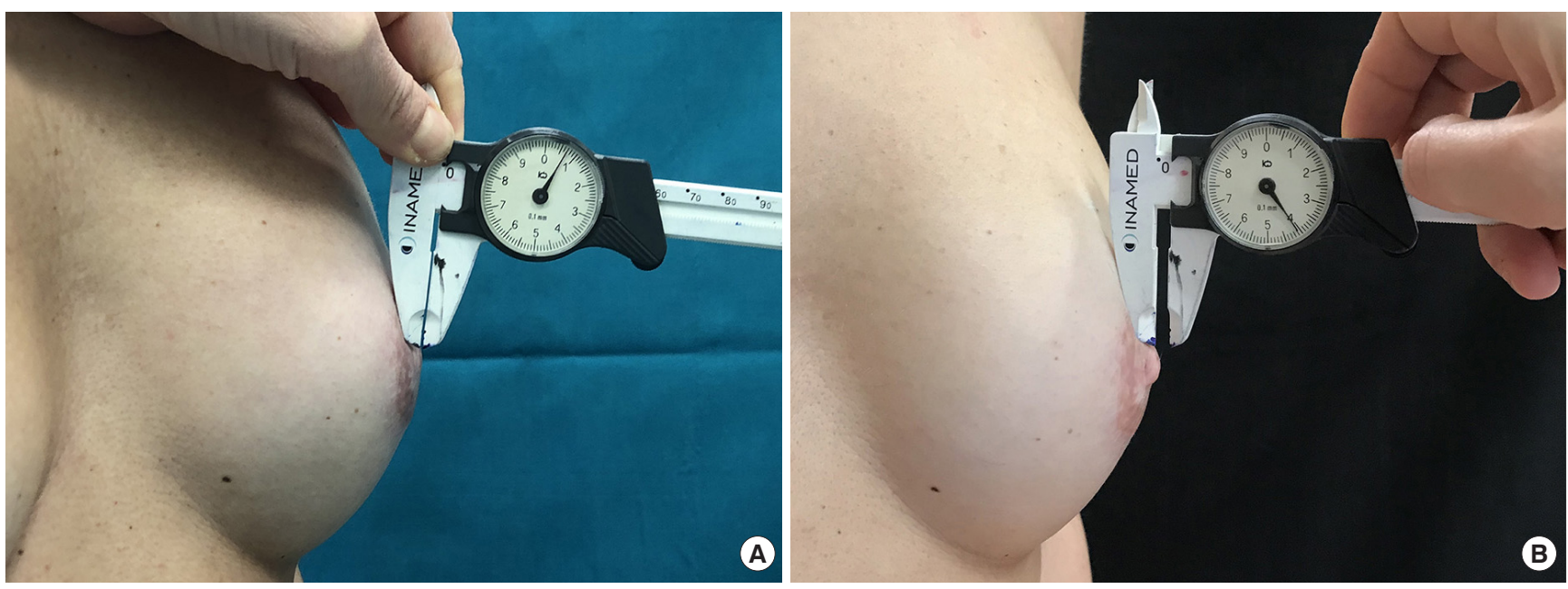

Fig. 5. Nipple reconstruction case with a graft. (A) Preoperative lateral view; nipple projection was measured with a caliber. (B) Postoperative lateral view at 12 months after the injection of Integra Flowable Wound Matrix.

We chose IFWM because we needed a material that is injectable but at the same time structured, enabling us to project a fully intact nipple through an outpatient procedure [14].

Twenty disease-free breast cancer patients were enrolled in our retrospective study. Their mean age was 51 years (range, 34-71 years). The average time window between reconstruction of the nipple by means of a graft or $\mathrm{C}-\mathrm{V}$ dermal flap and the injection of IFWM was 18 months (range, 6-38 months). The average follow-up after injection was 12 months.

The average measured projection of the previously reconstructed nipple before injection was $1.13 \pm 0.84 \mathrm{~mm}$ (range, $0-2.90 \mathrm{~mm}$ ), while the average projection was $4.76 \pm 0.88 \mathrm{~mm}$ (range, $4-8.23 \mathrm{~mm}$ ) at $\mathrm{P} 0$ and $4.23 \pm 0.42 \mathrm{~mm}$ (range, $3.70-5$ $\mathrm{mm}$ ) at both P6 and P12 (Fig. 2).

Between P0 and P12, the average projection increased by 3.1 $\mathrm{mm}$. The minimum increase in projection was $1 \mathrm{~mm}$ and the maximum increase was $5.1 \mathrm{~mm}$. No complications occurred at any time during follow-up, and the satisfaction of the patients (assessed through a visual analogue scale) averaged 4.25 out of 5 (Figs. 3-5).

Our results are in accordance with the existing literature on this topic [15]. We obtained a significant increase of nipple projection; specifically, the projection at 12 months after the injection of IFWM within the nipple was about four times $(4.23 \mathrm{~mm}$ on average) the preoperative projection (1.13 $\mathrm{mm}$ on average).

The very slight change in nipple projection between P0 (mean nipple projection: $4.76 \mathrm{~mm}$ ) and P12 (mean nipple projection: $4.23 \mathrm{~mm}$ ) led us to conclude that the effect of the injected IFWM on nipple projection remained stable over time.

A quick and simple procedure without any complications thus represents a source of great satisfaction for women who undergo life-saving breast surgery. Combined with local flaps or grafts for nipple reconstruction, IFMW appears to be a reliable technique for achieving nipple projection with long-lasting maintenance that was highly appreciated by patients as a single-step, minimally invasive, painless procedure with no reported necessity of reintervention.

\section{NOTES}

\section{Conflict of interest}

No potential conflict of interest relevant to this article was reported.

\section{Ethical approval}

All the procedures performed in studies involving human participants were in accordance with the ethical standards of the institutional and national research committee and with the 1964 Helsinki Declaration and its later amendments or comparable ethical standards. Written consent was achieved from all the participants in this study regarding the risk of complication, right to discontinue treatment.

\section{Patient consent}

The patients provided written informed consent for the publication and the use of their images.

\section{Author contribution}

Conceptualization: all authors. Data curation: S Tanini, S Calabrese. Formal analysis: S Tanini, S Calabrese, E Lucattelli. Methodology: S Tanini, G Lo Russo. Project administration: G Lo Russo. Visualization: all authors. Writing - original draft: all 
authors. Writing - review \& editing: all authors. Approval of final manuscript: all authors.

\section{ORCID}

Sara Tanini https://orcid.org/0000-0001-9749-1640

Sara Calabrese

Elena Lucattelli https://orcid.org/0000-0001-9066-0704

Giulia Lo Russo https://orcid.org/0000-0002-1501-8022 https://orcid.org/0000-0002-4800-9464

\section{REFERENCES}

1. Wellisch DK, Schain WS, Noone RB, et al. The psychological contribution of nipple addition in breast reconstruction. Plast Reconstr Surg 1987;80:699-704.

2. Momoh AO, Colakoglu S, de Blacam C, et al. The impact of nipple reconstruction on patient satis-faction in breast reconstruction. Ann Plast Surg 2012;69:389-93.

3. Gougoutas AJ, Said HK, Um G, et al. Nipple-areola complex reconstruction. Plast Reconstr Surg 2018;141:404e416 e.

4. Fansa H, Heitmann C. Reconstruction of the nipple areola complex (NAC). In: Fansa H, Heitmann C, editors. Breast reconstruction with autologous tissue. Cham: Springer; 2019. p. $175-80$.

5. Nahabedian MY. Nipple reconstruction. Clin Plast Surg 2007;34:131-7.

6. Jalini L, Lund J, Hornsby J, et al. Nipple reconstruction using the $\mathrm{C}-\mathrm{V}$ flap technique: long term outcome and patient satisfaction in a District General Hospital. Eur J Surg Oncol 2016;42:S49-50.
7. Shestak KC, Gabriel A, Landecker A, et al. Assessment of long-term nipple projection: a compari-son of three techniques. Plast Reconstr Surg 2002;110:780-6.

8. Winocour S, Saksena A, Oh C, et al. A systematic review of comparison of autologous, allogeneic, and synthetic augmentation grafts in nipple reconstruction. Plast Reconstr Surg 2016; 137:14e-23e.

9. Bernard RW, Beran SJ. Autologous fat graft in nipple reconstruction. Plast Reconstr Surg 2003; 112:964-8.

10. Park GY, Yoon ES, Cho HE, et al. Acellular dermal matrix as a core strut for projection in nipple reconstruction: approaches for three different methods of breast reconstruction. Arch Plast Surg 2016;43:424-9.

11. Panettiere P, Marchetti L, Accorsi D. Filler injection enhances the projection of the reconstructed nipple: an original easy technique. Aesthetic Plast Surg 2005;29:287-94.

12. Sisti A, Grimaldi L, Tassinari J, et al. Nipple-areola complex reconstruction techniques: a literature review. Eur J Surg Oncol 2016;42:441-65.

13. Campitiello F, Mancone M, Della Corte A, et al. Acellular flowable matrix in the treatment of tun-neled or cavity ulcers in diabetic feet: a preliminary report. Adv Skin Wound Care 2018;31:270-5.

14. Truong AT, Kowal-Vern A, Latenser BA, et al. Comparison of dermal substitutes in wound heal-ing utilizing a nude mouse model. J Burns Wounds 2005;4:e4.

15. Serre A, Guillier D, Moris V, et al. Nipple projection augmentation in breast reconstruction by arti-ficial derm injection. Ann Chir Plast Esthet 2017;62:625-9. 